\title{
Design and engineering of a simplified workflow execution for the MG5aMC event generator on GPUs and vector CPUs
}

\author{
Andrea Valassi $^{1, *}$, Stefan Roiser $^{1}$, Olivier Mattelaer ${ }^{2}$, and Stephan Hageboeck ${ }^{1}$ \\ ${ }^{1}$ CERN, IT-SC group, Geneva, Switzerland \\ ${ }^{2}$ Université Catholique de Louvain, Belgium
}

\begin{abstract}
Physics event generators are essential components of the data analysis software chain of high energy physics experiments, and important consumers of their CPU resources. Improving the software performance of these packages on modern hardware architectures, such as those deployed at HPC centers, is essential in view of the upcoming HL-LHC physics programme. In this paper, we describe an ongoing activity to reengineer the Madgraph5_aMC@NLO physics event generator, primarily to port it and allow its efficient execution on GPUs, but also to modernize it and optimize its performance on vector CPUs. We describe the motivation, engineering process and software architecture design of our developments, as well as the current challenges and future directions for this project. This paper is based on our submission to vCHEP2021 in March 2021, complemented with a few preliminary results that we presented during the conference. Further details and updated results will be given in later publications.
\end{abstract}

\section{Introduction}

MadGraph5_aMC@NLO [1] (in the following, MG5aMC) is a physics event generator software used in the data processing workflows of High Energy Physics (HEP) experiments, such as ATLAS and CMS at CERN's Large Hadron Collider (LHC). While the majority of CPU resources in the distributed computing environments of the LHC experiments is spent on detector simulation and event reconstruction workflows, physics event generators are also large consumers of CPU time, accounting for an estimated $12 \%$ of overall CPU budgets for ATLAS and $5 \%$ for CMS [2]. Their computational cost is also predicted to increase during the HighLuminosity phase of LHC (HL-LHC), as more accurate theoretical predictions are required for physics analyses. Reengineering event generators to improve their software performance in view of the HL-LHC, especially on modern hardware architectures such as CPUs with many cores and wide vector registers, is therefore essential $[2,3]$.

With wider availability of graphics processing units (GPUs) for computing, the question also arises about whether event generators like MG5aMC may efficiently exploit these new architectures. In recent years, many high performance computing centers (HPCs) have deployed heterogeneous systems, where the majority of the computing power is provided by GPUs. These resources are currently under-utilized by HEP experiments, also because the port to GPUs of other HEP offline Grid workflows, such as detector simulation, has not yet been achieved. Porting event generators to GPUs would therefore be especially important.

The current production version of the MG5aMC software has been developed for CPUs only. Previous work for porting some of its components to CUDA [4] on Nvidia GPUs was done around 10 years ago [5-9], but unfortunately never reached production quality. This effort has been restarted in early 2020 by the authors of this paper (including one of main authors of the MG5aMC software), eventually as part of the activities of a larger team. The project [10] is a collaboration between theorists, experimentalists and software engineers, which largely came about through the activities of the HSF Physics Event Generator WG [11]. This paper describes our software development process and engineering work for the port of

\footnotetext{
*e-mail: andrea.valassi@cern.ch
} 
MG5aMC to GPUs, and more specifically on Nvidia/CUDA, and for efficiently exploiting vector CPUs by an improved $\mathrm{C}++$ implementation. Our colleagues in the larger development team are also working on a port to GPU architectures from other hardware vendors, notably through the use of abstraction layers, but this work will eventually be described elsewhere. While our development focuses on MG5aMC, we believe that many of the ideas and methods we use may also be useful for the software reengineering of other event generators.

This paper is based on our March 2021 submission to the vCHEP2021 [12] conference, complemented with some preliminary results that we presented [13] there. Its structure is the following. We provide a brief description of the MG5aMC code in Sec. 2. An overview of the engineering process is given in Sec. 3. We describe the high-level software architecture design, some implementation details and work in progress in Sec. 4. Our preliminary results and future plans are summarized in Sec. 5. We give our interim conclusions in Sec. 6. Further details and updated results for this ongoing activity will be presented in later publications.

\section{Brief description of the MG5aMC software}

MadGraph5_aMC@NLO (MG5aMC) [1] is a computer program which aims at providing in a unified framework all the elements necessary for the phenomenological study of a wide spectrum of HEP collision processes, both within the Standard Model (SM) and beyond (BSM). In particular, MG5aMC allows the computation of cross sections and generation of hard events at tree level (LO) and next-to-leading order (NLO), their matching [14] to parton shower (PS) simulations, and the merging of matched samples that differ by the number of jets (i.e. gluons and light quarks) in the final state. Only a subset of these many features are relevant to the work presented in this paper, which is presently focusing on simple LO processes.

The general techniques used in MG5aMC to integrate a LO partonic cross section, and to obtain a set of unweighted events from it, have been inherited from MadGraph5 [15]. This strategy has then undergone successive optimisations, including some very recent ones [16]. The core of the process is the calculation of tree-level invariant amplitudes and "matrix elements" (ME) from the set of all relevant Feynman diagrams. Since this depends on the collision process of interest, MG5aMC is constructed as a Python meta-code, which is capable of automatically generating the ME code specific to the desired process. The Lagrangian of the physics model can be expressed in a Python based format, the Universal FeynRules Output (UFO) [17], thanks to various codes such as FeynRules [18], LanHep [19] and Sarah [20]. Taking a given process and UFO as inputs, MG5aMC determines the set of relevant Feynman diagrams and generates the code to evaluate the associated transition amplitude for an arbitrary phase space point. Thanks to the flexibility of Python, the code can be generated in various programming languages: Fortran is the default, but $\mathrm{C}++$ and Python are supported as well. The phase space points where the ME calculation functions are evaluated are determined by a Monte Carlo (MC) integrator, using a sampling algorithm based on random number generation. The integrator is the component that drives the whole process of event generation, cross section integration and event unweighting: in the production version of MG5aMC, this is the Fortran package MadEvent, which uses an optimized phase space sampling algorithm based on single diagram enhancement [16, 21].

The calculation of matrix elements from Feynman diagrams in MG5aMC is based on helicity amplitudes [22-25] and QCD (Quantum Chromo Dynamics) color-flow decomposition [26]. With respect to trace techniques based on completeness relations [27], the main advantage of this method is that interference terms are handled by a simple sum over amplitudes and, therefore, the computational complexity grows linearly with the number of diagrams instead of quadratically. In practice, amplitudes (which are complex numbers) for all diagrams are computed numerically for a given combination of helicities of the external (initial and final state) particles, and are then organized into subsets, each corresponding to a given QCD color flow (i.e. a given combination of colors) of the external particles. The amplitudes 

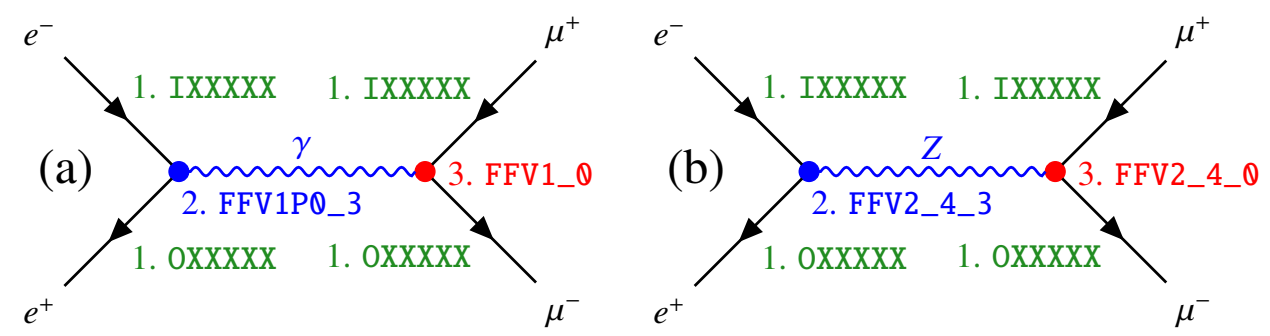

Figure 1. The two Feynman diagrams contributing to $e^{+} e^{-} \rightarrow \mu^{+} \mu^{-}$at tree level: (a) photon exchange and (b) $\mathrm{Z}$ exchange. The routines called by MG5aMC to compute the ME for each event are also indicated. First, the wavefunctions of the four external fermions are computed using IXXXXX and OXXXXX, given their momenta and helicities. This is only done once, as the result is the same for both diagrams. Next, the wavefunctions of the $\gamma$ and $Z$ propagators in the two diagrams are computed from their coupling to the initial state electrons, using FFV1PO_3 and FFV2_4_3. Finally, the transition amplitudes are computed from the boson couplings to the final state muons in the two diagrams, using FFV1_Q and FFV2_4_0. The two amplitudes are added to each other, their sum is squared, and the result is averaged over all valid helicity combinations to obtain the matrix element. Each of the four fermions has two possible helicity states, therefore there are in principle sixteen helicity combinations; however, as the $\gamma$ and $Z$ are vector bosons with spin 1, only four of those are allowed. Drawn with TikZ-Feynman [28].

associated with different diagrams for a color flow are summed to give the so called dual amplitude for that color flow. The squared amplitude for a given helicity combination is then computed as a quadratic form on the vector of the dual amplitudes for different color flows, using a pre-computed color matrix, which is the same for all events. Finally, the average of these squared amplitudes over all helicity combinations yields the overall matrix element for the collision event. In this last step, only a specific subset of helicity combinations have a non-zero contribution to the $\mathrm{ME}$, as most combinations are forbidden by the conservation of angular momentum; to speed up the computation for large samples of events, MG5aMC pre-determines which helicity combinations are allowed by calculating their contributions on a small set of events. In the example of the $e^{+} e^{-} \rightarrow \mu^{+} \mu^{-}$collision shown in Fig. 1, sixteen different helicity combinations exist, as there are four external fermions with two possible spin polarizations each, but only four of those combinations are allowed by conservation laws.

The transition amplitude for a given process is computed in MG5aMC using the helicity amplitude routines provided by the sub-program ALOHA [25]. Those routines are almost identical to the ones of the HELAS library [29, 30], which was used in MadGraph4. While HELAS functions are all hardcoded, ALOHA generates most of the required functions dynamically. The main advantage of this approach, adopted in the MadGraph5 [15] release, is that it is not limited to predetermined physics models, and can be easily extended. This is also essential in the specific work presented in this paper, as it makes it easier to port and optimize the software for new GPU and CPU architectures directly in the MG5aMC code base, without relying on changes to external libraries. A distinctive feature of helicity amplitude methods is that each particle in a Feynman diagram is associated to a "wavefunction" that contains its spinorial representation: this is a vector of complex numbers whose dimensions depend on the properties of each particle (essentially, on its spin). For instance, as in HELAS [29], the wavefunction for a fermion in ALOHA is a vector of six complex numbers: two for the chirality-left spinor, two for the chirality-right spinor, and two for its 4-momentum. In the helicity wavefunctions formalism, the computation of the ME code is performed thanks to three categories of routines used in a sequential way, as shown in Fig. 1 (and later in Fig. 3). First, a wavefunction is computed for each initial or final state particle in the Feynman diagram, given its 4-momentum and helicity using an "external particle" routine (e.g. IXXXXX for the ingoing and OXXXXX for the outgoing electrons and muons in Fig. 1). Second, for each vertex 
in the Feynman diagram which has one leg with no associated wavefunction, this is computed using an "internal particle" routine (e.g. FFV1PQ_3 for the off-shell propagator at the electron-electron-photon vertex in Fig. 1). Finally, when the wavefunctions for all the legs at a vertex are known, the amplitude for the diagram is computed using an "amplitude" routine (e.g. FFV1_Q for the muon-muon-photon vertex in Fig. 1). More details on this formalism and on the various optimizations implemented within MG5aMC are given in Ref. [16].

\section{Software engineering process}

From an engineering perspective, MG5aMC is a code generator, written in Python. Given a physics process, it generates the corresponding process-specific source code in multiple implementation languages. The source code will then be compiled into an executable to produce the desired physics calculations. At the time of writing, MG5aMC provides code back-ends to produce Fortran, $\mathrm{C}++$ and Python source code. Fortran is the only backend used in production by the LHC experiments.

\section{Iterative engineering in $C U D A / C++$}

As described in Sec. 1, the primary goal of this project is to add one or more back-end implementation languages that will allow the workflow execution also on graphics cards from various vendors such as Nvidia, AMD or Intel. In this paper, we focus on the engineering for Nvidia GPUs with the $\mathrm{C} / \mathrm{C}++$ flavor of CUDA. In the future, we foresee the possibility to support AMD GPUs using the HIP [31] language. Other members of our team are also investigating hardware abstraction layers such as Alpaka [32], Kokkos [33] and SYCL [34], which allow one to write a single implementation that may be executed on various GPU (and CPU) hardware back-ends.

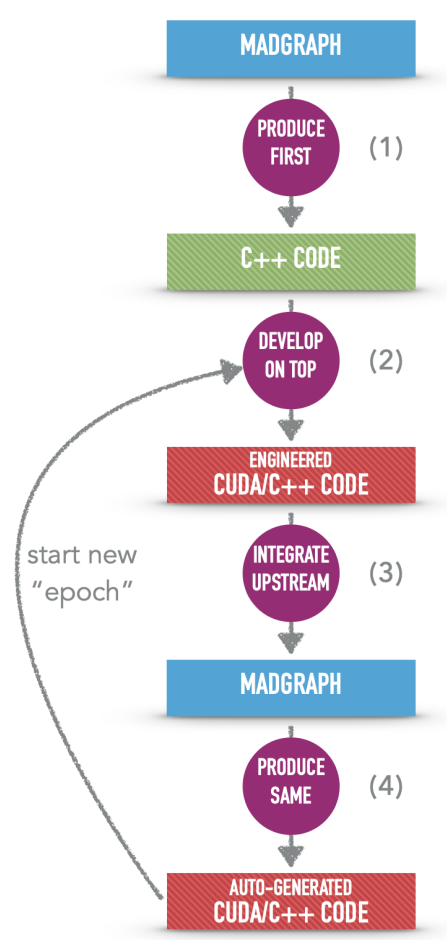

Figure 2. The overall software engineering process is iterative.

A complementary goal for the project, which came about naturally during our development in the $\mathrm{C} / \mathrm{C}++$ flavor of CUDA, is the reengineering and performance optimization of the $\mathrm{C}++$ back-end of MG5aMC for CPUs. Because of the large overlap between the two backends, we use a single source code implementation for CUDA and $\mathrm{C}++$, where more than $90 \%$ of the code is shared, and a few \#ifdef's enclose the remaining back-end-specific code.

The engineering towards the CUDA/C++ implementation is designed around a cycle of developing and optimizing software in CUDA/C ++ and back-porting this upstream into the MG5aMC Python code generator. In the bootstrapping phase, the $\mathrm{C}++$ back-end was used to generate the source code of the $e^{+} e^{-} \rightarrow \mu^{+} \mu^{-}$physics process (see Fig. 2, step 1) in a so called "epoch0". This code was used as the basis to engineer a first CUDA/C++ version (Fig. 2, step 2), which was subsequently integrated upstream into MG5aMC (Fig. 2, step 3) to autore-generate the same CUDA/C++ code (Fig. 2, step 4), with a new "epoch1" engineering step starting. The newly produced code served as the basis towards the engineering of a new "epoch2" in steps 2-4. The results we present in Secs. 4-5 are based on "epoch2". At the time of writing, we are back-porting $\mathrm{C}++$ vectorization into the code generator, which we will then use to produce a new "epoch3". The code we develop and additional work on abstraction layers are hosted on github [35]. The code version described in this paper was also tagged and published on Zenodo [36]. The official MG5aMC repository, where our work is backported to the Python meta-code whenever necessary, continues to be hosted on launchpad [37]. 


\begin{tabular}{lrrr}
\hline Process & LOC & functions & function calls \\
\hline$e^{+} e^{-} \rightarrow \mu^{+} \mu^{-}$ & 776 & 8 & 16 \\
$g g \rightarrow t \bar{t}$ & 839 & 10 & 22 \\
$g g \rightarrow t \bar{t} g$ & 1082 & 36 & 106 \\
$g g \rightarrow t \bar{t} g g$ & 1985 & 222 & 786 \\
\hline
\end{tabular}

Table 1. Comparison of the complexity of generated CUDA code for various physics processes, as the number of relevant Feynman diagrams increases. The columns represent the number of auto-generated lines $(L O C)$, the number of individual generated functions (functions) and the number of function calls during one calculation (function calls), as several functions are called multiple times with different parameters. An additional source of complexity, not shown in this table or in Fig. 3, is the size of the color matrix required by the QCD color flow algebra, described in Sec. 2.

Although the process $e^{+} e^{-} \rightarrow \mu^{+} \mu^{-}$is not relevant for LHC physics, we chose it as a starting point for CUDA implementations in order to simplify the engineering work itself, as it involves only a limited number of lines of CUDA/C++ code to work on in each epoch. With each back-port of the code into MG5aMC for a new epoch, we also generate source code for more complex QCD processes such as $g g \rightarrow t \bar{t}, g g \rightarrow t \bar{t} g$ and $g g \rightarrow t \bar{t} g g$. This is used to validate the code generator and as a basis for further optimizations, more directly relevant to LHC physics. The increase in complexity of the auto-generated code for the four processes considered is shown in Tab. 1 and Fig. 3, for the ME calculation part of the workflow.

\section{Simplified workflow application - performance optimization and physics validation}

The ME calculation is the core component of MG5aMC not only from a physics point of view, but also and more importantly because it is by far the largest consumer of CPU in the overall workflow. In a MG5aMC Fortran executable for $g g \rightarrow t \bar{t} g g$ events, the ME calculation accounts for more than $90 \%$ of the CPU instructions, and this fraction further increases for more complex LHC physics processes [16].
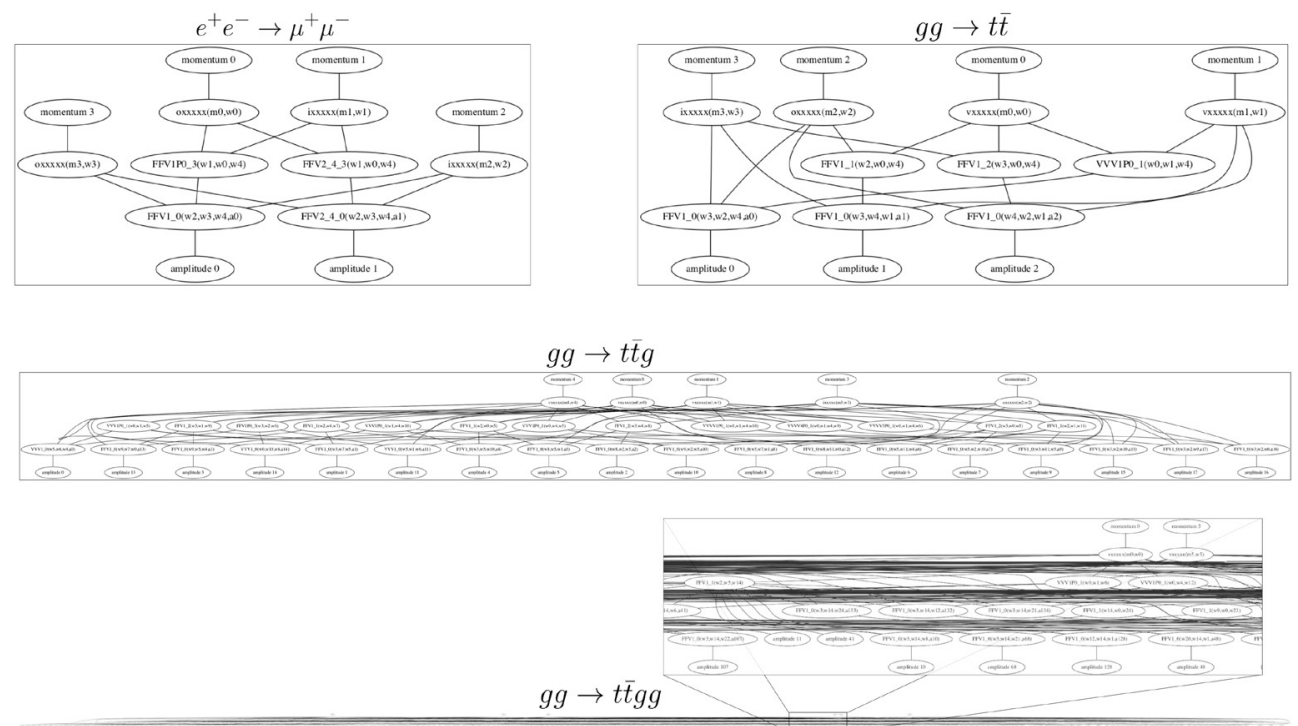

Figure 3. Visualization of the complexity of ME calculations for various physics processes. The detailed computation algorithm is described in Sec. 2. The only inputs to the calculation which vary from one event to another are the 4-momenta of the initial and final state particles in the collision (4 for $e^{+} e^{-} \rightarrow \mu^{+} \mu^{-}$and $g g \rightarrow t \bar{t}, 5$ for $g g \rightarrow t \bar{t} g$ and 6 for $g g \rightarrow t \bar{t} g g$ ). 


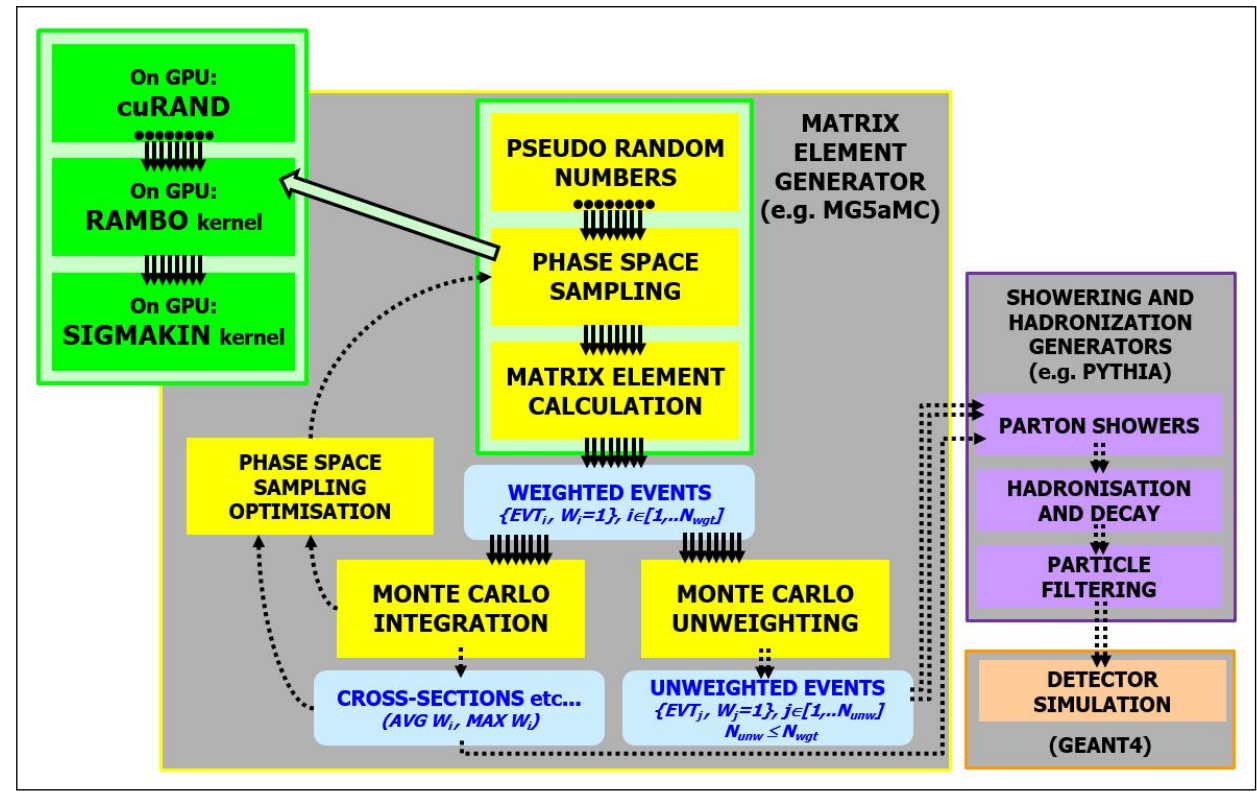

Figure 4. Schematic representation of the computational anatomy of a matrix element generator. The three green blocks in the top-left corner represent the software components included in the standalone simplified workflow application described in this paper. A detailed explanation is provided in the text.

Our development so far, therefore, has mainly focused on optimising the CUDA and C++ software performance of the ME calculation within a simplified workflow application, rather than on implementing a CUDA/C ++ framework providing the full functionality of MG5aMC. As described in Sec. 4 and Fig. 4, our standalone application includes the generation of random numbers for a given number of events using the Nvidia cuRAND [38] library, their mapping to 4-momenta of the particles in each collision using a simple phase space sampling algorithm, RAMBO [39], and the ME calculation for each event from these 4-momenta.

The level of functional testing that we have deemed appropriate for this simple workflow is minimal. The output of our "toy" application includes both performance metrics and physics results, which are visually inspected when running it after a code change. To validate physics correctness, the average of the MEs over all processed events is compared to an expected value. A bitwise comparison is possible if the same random number seeds and event sample sizes are used, as the average ME is strictly reproducible in this case, and even yields the same result in CUDA and $\mathrm{C}++$ as we use a cuRAND algorithm generating the same random sequences on GPU and CPU; in all other cases, the average ME result is checked within its statistical error. In general, the average ME is not a physics relevant quantity per se, but it is enough for a basic check of the correctness of physics results; in the future this will be complemented by the proper computation of fiducial cross sections. In addition, functional tests based on the GoogleTest [40] API have been developed to compare MEs and particle 4-momenta of several individual events against pre-computed references, for both CPU and GPU; these tests are also executed in a continuous integration in github.

\section{Software architecture design and implementation challenges}

\section{Component decomposition of Matrix Element Generators — event-level data parallelism}

The very first step in our reengineering of MG5aMC, and in the design of a software architecture enabling efficient parallel processing on both GPUs and CPUs, was to deconstruct its computational workflow into separate components and to identify their input and output 
data. This phase of the project, which overlapped with the work ongoing in the HSF Event Generator WG for preparing the LHCC review of HL-LHC computing [2, 3], was also key to enabling the collaboration between people with diverse skills and backgrounds, by translating theoretical physics methodologies into practical computational problems.

As described more in detail in Refs. [2, 3], a Matrix Element Generator (MEG) like MG5aMC is a computer program that, starting from a limited set of input configuration parameters, is typically used to produce two types of outputs: a fiducial cross section for the collision process and a sample of $N_{\text {unw }}$ unweighted events. Both results are obtained using Monte Carlo methods, starting from a larger set of $N_{\text {wgt }}$ randomly generated weighted events. Each event is described by the 4-momenta of all initial and final state particles, and by an event weight, which varies for weighted events, but is a constant equal to +1 for unweighted events. The resulting sample of "parton-level" unweighted events and the process cross section are written out to a file (typically using the Les Houches Event File, or LHEF [41, 42], format), which is then read back as an input by a Showering and Hadronization Generator (SHG) like Pythia [43]. In turn, the SHG adds parton showers, hadronisation and particle decay to each event, filters out events based on some selection criteria, and writes the resulting sample of "particle-level" events to a file. This file is then read as an input by the detector simulation framework Geant4 [44], which produces yet another output for further event processing components down the line. This description of a typical data flow is represented schematically in Fig. 4. It does not cover all possible use cases, some of which may be significantly more complex (and involve, for instance, jet matching and merging, or unweighted events with negative weights), but it is enough for the collision processes that were used in this project.

The core of the MEG computation is the preparation of the sample of weighted events. This involves three steps, represented by the green boxes in Fig. 4. First, a set of random numbers is drawn for each event using a pseudo-random number generator. Starting from these, the particle 4-momenta in each event, as well as a sampling weight, are then constructed by a phase space sampling algorithm. The event weight is finally derived by combining the sampling weight with the matrix element, which is computed starting from these 4-momenta.

The key feature of this computational workflow is that the numerical operations transforming input data into output data, in the phase space sampling algorithm and in the ME calculation, are exactly the same for all of the events (or, at least, for all of the events within the same subprocess of the overall collision process). This makes a MEG like MG5aMC an ideal candidate to implement data parallelism using lockstep processing on GPUs and vectorized code on CPUs. As a consequence, the engineering work in this project relies, first and foremost, on a software design based on event-level data parallelism: the basic idea is to translate each and every data processing step into a stateless function that operates simultaneously on a basket of events, rather than on an individual event. This is especially important for the ME calculation, which (for all but the simplest physics processes) is the computational hot-spot of the whole workflow. A crucial aspect of MEGs is that random numbers are mainly used for Monte Carlo sampling, rather than to take Monte Carlo decisions. In other words, conditional branching of a stochastic nature does exist in MEGS, but only in a few well-defined situations (such as event filtering due to phase space cuts, and event unweighting), where data stream compaction should in principle be straight-forward and have a computational cost that is relatively negligible in comparison to that of ME calculations.

\section{High-level design of software components and data structures}

In practice, one of the most important aspects in the software architecture of a data parallel system is the design of the relevant data structures. As mentioned above, our toy application includes three main components: a random number generator, a phase space sampler, and a ME calculation engine. The interface of each component is designed to operate on multiple events in parallel; rather than using $\mathrm{C}++$ vectors, the relevant inputs and outputs are read 

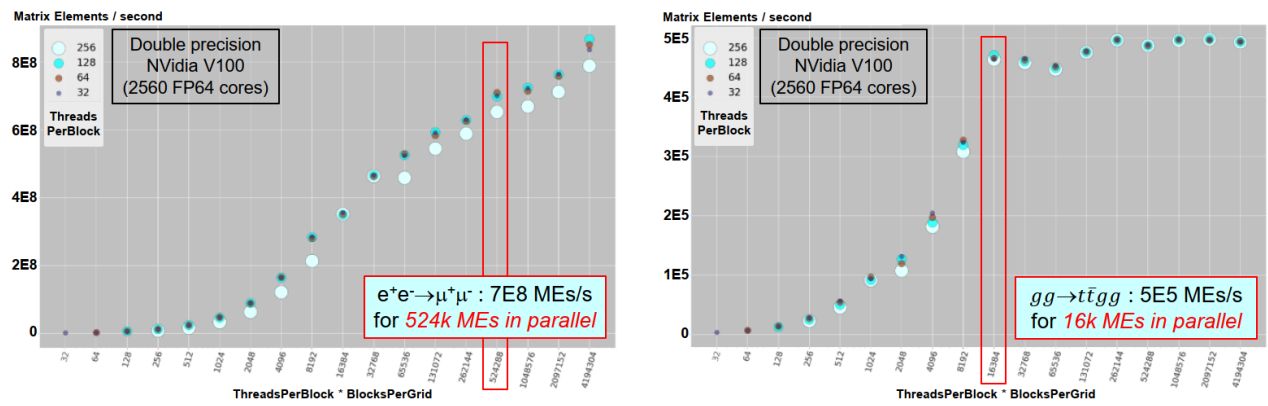

Figure 5. Throughput (MEs per second) of the ME calculation in CUDA, as a function of the number of threads (i.e. the number of events processed in one iteration), using double precision floating point arithmetics, for $e^{+} e^{-} \rightarrow \mu^{+} \mu^{-}$(left) and $g g \rightarrow t \bar{t} g g$ (right). Four sets of results are plotted for different values of the number of threads per block. Throughputs are computed as the number of MEs divided by the sum of the time to compute them on the GPU device and of the time to copy them to the CPU host. Virtual machine itscrd70 with an Nvidia V100 GPU; details of the software build are given in Tab. 2. For illustrative purposes only, the red boxes in the two plots indicate two examples discussed in the text.

from and written into C-style arrays with fixed dimensions, which are properly sized to contain a pre-defined number of events that is meant to be processed in one "iteration". In our single-source code approach, similar memory layouts are used for CUDA and C++, but many specific choices differ in the two implementations, and are in any case highly configurable. It is also worth noting that CUDA and $\mathrm{C}++$ modules are built in slightly different ways, as we observed that the CUDA implementation is faster when Relocatable Device Code is disabled.

In the CUDA implementation, each event is processed by one GPU thread. Thus, the number of events in one iteration corresponds to the number of threads in a "grid", i.e. to the product of the numbers of "blocks" per grid and of threads per block, which together with the number of iterations are configured by three command-line user inputs. For $e^{+} e^{-} \rightarrow \mu^{+} \mu^{-}$, a typical measurement consists in processing $6 \mathrm{M}$ events in 12 iterations of $524 \mathrm{k}$ events, partitioned in 2048 blocks of 256 threads; in practice, one CUDA iteration in this case consists in generating all relevant random numbers (four per external particle) for 524k events using the cuRAND library, and in submitting over a grid of 524k threads a phase space sampling (RAMBO) kernel and a ME calculation (SIGMAKIN) kernel. This specific choice of parameters is due to the observation that the throughput of the ME calculation for $e^{+} e^{-} \rightarrow \mu^{+} \mu^{-}$on an Nvidia V100 [45] GPU only approaches its maximum plateau when $O(1 \mathrm{M})$ threads per grid are used, as shown in Fig. 5; for comparison, around 16k threads per grid are enough to reach the throughput plateau for $g g \rightarrow t \bar{t} g g$, as the ME calculation kernel for this process is much more computationally intensive. A few iterations are used to smooth out performance fluctuations, while still requiring less than one second overall for one throughput measurement for $e^{+} e^{-} \rightarrow \mu^{+} \mu^{-}$.

In the $\mathrm{C}++$ implementation, data processing is also split into iterations configured using the same three command line arguments. While the concept of CUDA thread blocks has no equivalent in $\mathrm{C}++$, this is used to configure some parameters (e.g. random number seeding in cuRAND) in the same way as in CUDA, to achieve reproducibility of physics results on an event-by-event basis between CUDA and $\mathrm{C}++$ when possible. A typical throughput measurement for generating $6 \mathrm{M}$ events over 12 iterations takes only a few seconds for $e^{+} e^{-} \rightarrow \mu^{+} \mu^{-}$.

Memory is allocated before the first iteration and released after the last one. Data structures are allocated where the computations take place, i.e. on the GPU device for CUDA and on the CPU host heap for $\mathrm{C}++$. In the CUDA implementation, 4-momenta, sampling weights and MEs are also heap-allocated on the CPU host, where they are copied after each iteration. This data transfer from GPU to CPU is slow and can add a large overhead to the ME computation, even if pinned host memory is used to speed it up. The ratio of ME copy time to ME computation time is approximately $1: 1$ for $e^{+} e^{-} \rightarrow \mu^{+} \mu^{-}$, but this decreases to 1:2500 for 


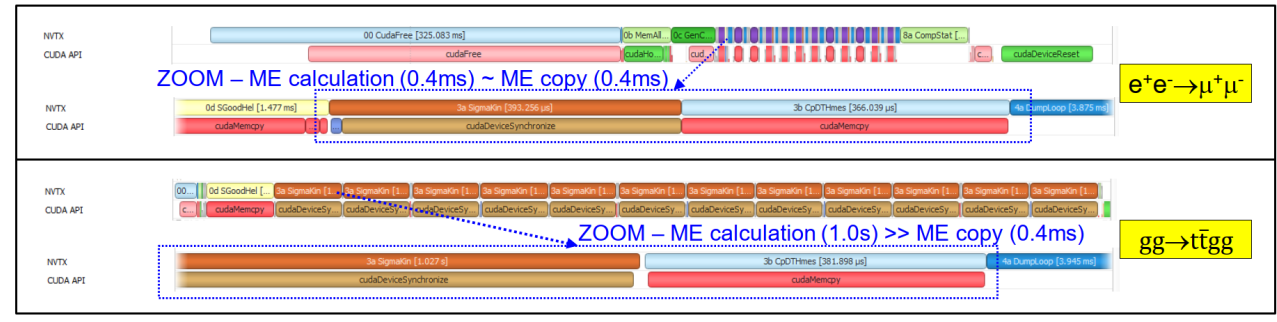

Figure 6. Visualization of the CUDA workflow using Nvidia Nsight Systems [46], for $e^{+} e^{-} \rightarrow \mu^{+} \mu^{-}$(top) and $g g \rightarrow t \bar{t} g g$ (bottom). Both sets of diagrams refer to the execution of the standalone executable, using 12 iterations with 524k events each (2048 blocks, 256 threads per block). The ME calculation takes about $0.4 \mathrm{~ms}$ and $1.0 \mathrm{~s}$ per iteration for $e^{+} e^{-} \rightarrow \mu^{+} \mu^{-}$and $g g \rightarrow t \bar{t} g g$, respectively, while the ME copy takes about $0.4 \mathrm{~ms}$ in both cases. Including both times in the computation of throughputs, this corresponds to processing rates of about $5 \mathrm{E} 5 \mathrm{MEs} / \mathrm{s}(5 \mathrm{E} 5 \mathrm{MEs} / 1.0 \mathrm{~s})$, and $7 \mathrm{E} 8 \mathrm{MEs} / \mathrm{s}$ (5E5MEs/0.8ms), respectively. Virtual machine itscrd70 with an Nvidia V100 GPU; details of the software build are given in Tab. 2.

$g g \rightarrow t \bar{t} g g$, as shown in Fig. 6. We therefore expect that the overhead of device-to-host data transfers should be completely negligible for the complex processes relevant to LHC physics.

The outputs of the cuRAND, RAMBO and SIGMAKIN steps, namely random numbers, 4-momenta and weights, are all arrays of floating point real numbers. Double precision is used by default throughout all internal calculations in both CUDA and $\mathrm{C}++$, to be consistent with the choice made in the FORTRAN code to achieve the required physics accuracy. At build time, one can also switch to single precision: while we initially added this feature to enable tests of consumer-grade GPUs, this turned out to be very useful to compare the double and single precision throughputs of the ME calculation, on different types of GPUs for data centers or using different levels of vectorization on CPUs, as discussed further below.

Several memory layouts were prototyped, including arrays of structures (AOS), structures of arrays (SOA), and arrays of structures of arrays (AOSOA). In this nomenclature, "structure" (S) reflects the data organization within each event, while "array" (A) indicates independent events. All layouts are coded as 1-dimensional C-style arrays, but are decoded using multi-dimensional indices. The AOSOA layout is now the default: data are partitioned in event "pages", where each page (the leading A) represents a different subset of events and is organized internally as an SOA. For instance, the 4-momenta AOSOA is logically a fourdimensional array, where the four indices run over the number of event pages, the number of particles in each event, the 4 components of 4-momenta, and the number of events per page.

The number of events per page, i.e. the striding of the AOSOA, is highly configurable and can differ in the CUDA and $\mathrm{C}++$ implementations and in the random number and 4-momenta arrays. As an example, the 4-momenta AOSOA in CUDA has 4 events per page in double precision and 8 in single precision: this choice ensures that that a given data item (e.g. the energy of the first particle) for different events in the same page is aligned so that it can be fetched in one 32-byte transaction [47] on a V100 GPU. Profiling using Nvidia Nsight Compute [48] confirms that this choice achieves coalesced memory access, i.e. it fulfills all data requests (11tex__t_requests_pipe_lsu_mem_global_op_ld.sum [49]) using a minimal number of memory transactions (11tex__t_sectors_pipe_lsu_mem_global_op_ld.sum [49]). With respect to AOS layouts (which can be recovered by using an AOSOA striding with one event per page), the optimal AOSOA layouts for the 4-momenta array reduce the number of memory transactions by a factor 4 in double precision (or a factor 8 in single precision). Using AOSOA's instead of AOS's, however, only increases the throughput of the ME calculation by approximately $5 \%$ to $10 \%$ : this is probably because, even in the simplest $e^{+} e^{-} \rightarrow \mu^{+} \mu^{-}$case, the cost of the calculation is much higher than that of data access from GPU global memory.

While the AOSOA layout was initially designed for the CUDA GPU implementation, and specifically for data transfers at the interface between the three main components of our 
simplified application, the benefits of this type of layout, where the variables representing the same data item for different events are adjacent to one another, turned out to be especially important in the $\mathrm{C}++$ implementation, and more specifically in the internal implementation of the SIGMAKIN component, where this represents an essential and unavoidable ingredient of vectorization. This is described in more detail in the following paragraph.

\section{Optimising ME calculations: CUDA register pressure, $C++$ vectorization and multithreading}

The SIGMAKIN component, where matrix elements are calculated, is the computational hotspot of the application. For this reason, this is the component where most of our optimisation efforts have focused so far, and where we plan to invest more work to obtain further performance improvements in the future, in both the CUDA and $\mathrm{C}++$ implementations.

As described in Sec. 2, the ME calculation follows the helicity amplitude formalism; this involves a few IXXXXX and OXXXXX calls and many calls to "FFV" functions like FFV1_0, all of which operate on particle wavefunctions using complex number arithmetics. To ensure portability between CUDA and $\mathrm{C}++$, as well as to implement vectorization in $\mathrm{C}++$, we use a custom definition of complex number types, which is a light wrapper over std: : complex for $\mathrm{C}++$ and thrust : : complex (or. optionally, cuComplex) for CUDA. Double or single precision complex numbers are used, depending on the type chosen for 4-momenta and MEs.

In our current CUDA implementation, SIGMAKIN is a single kernel. In practice, this encapsulates all of the function calls in one of the diagrams in Fig. 3. The amount of data that this kernel needs to process is therefore quite large, as it includes the wavefunctions that the FFV functions exchange with one another, as well as the data that each FFV function needs internally. This is potentially a problem, especially for complex processes involving a large number of Feynman diagrams and FFV functions, because each CUDA thread can only access a limited number of registers [50] (e.g. 255 on a V100), and using more data than fits in registers leads to spilling to global memory. Already in the simple $e^{+} e^{-} \rightarrow \mu^{+} \mu^{-}$process, we have observed that "register pressure" limits the performance of our code: according to Nsight Compute (launch__registers_per_thread), SIGMAKIN uses 120 registers in double and 48 in single precision on a V100. One algorithmic improvement that has resulted in significant increases in ME throughputs (up to 2x in CUDA and 1.3x in $\mathrm{C}++$ for $e^{+} e^{-} \rightarrow \mu^{+} \mu^{-}$), which for CUDA can partly be explained in terms of reduced register pressure, has consisted in implementing simpler versions of the IXXXXX and OXXXXX functions, for specific use cases (e.g. if a particle mass or transverse momentum is 0 ). Another, more limited, speedup was obtained by storing physics model parameters (like masses and couplings) in CUDA constant memory. We have also done a few initial tests using shared memory to hold the intermediate wavefunctions, but a more comprehensive approach is needed. In particular, one interesting option would consist in splitting the large SIGMAKIN kernel into a number of smaller, independent kernels (e.g. one kernel per FFV call), possibly orchestrating the process using CUDA Graphs [51]; this will certainly be an active area of our development in the future.

To reduce register pressure, we are also considering to try a radically different way to achieve lockstep, by using different GPU threads to process different helicities for the same event. For the time being, however, our default approach to achieve lockstep processing remains event-level parallelism, where a different event is assigned to each CUDA thread. So far, in fact, this fundamental design choice has been a success. Implementing this strategy was relatively easy and did not require major preparatory work: as mentioned, AOSOA layouts do provide a benefit for CUDA memory access, but strictly speaking they are not essential. Profiling our code using Nvidia Nsight Compute indicates that the SIGMAKIN kernel reaches a $100 \%$ branch efficiency (sm__sass_average_branch_targets_threads_uniform.pct [49]), i.e. does not exhibit thread divergence. In other words, event-level parallelism in our code does efficiently exploit the SIMT (Single Instruction Multiple Threads) [52] architecture of the GPU, as the same instructions are executed in lockstep by the CUDA threads in a warp. 


\begin{tabular}{|c|c|c|c|c|}
\hline \multirow{2}{*}{$\begin{array}{l}\text { CPU build name }\left(e^{+} e^{-} \rightarrow \mu^{+} \mu^{-}\right) \quad \text { (SIMD spec) } \\
\text { [SIMD compiler flags] }\end{array}$} & \multirow{2}{*}{$\begin{array}{l}\text { \#doubles } \\
\text { per vector }\end{array}$} & \multirow{2}{*}{$\begin{array}{l}\text { \#floats } \\
\text { per vector }\end{array}$} & \multicolumn{2}{|c|}{ MEs/sec (compute) } \\
\hline & & & Double & Float \\
\hline $\begin{array}{l}\text { MadEvent Fortran } \\
{[-]}\end{array}$ & $\mathrm{x} 1$ & $\mathrm{x} 1$ & $\begin{array}{l}1.50 \mathrm{E} 6 \\
(\mathrm{x} 1.15)\end{array}$ & - \\
\hline $\begin{array}{l}\text { Standalone } \mathrm{C}++ \text { "none" } \\
{[-]}\end{array}$ & $\mathrm{x} 1$ & $\mathrm{x} 1$ & $\begin{array}{l}1.31 \mathrm{E} 6 \\
\mathbf{( x 1 . 0 0 )}\end{array}$ & $\begin{array}{c}1.21 \mathrm{E} 6 \\
(\mathrm{x} 0.92)[x 1.00]\end{array}$ \\
\hline $\begin{array}{l}\text { Standalone C++"sse4" } \\
{[- \text { march=nehalem }]}\end{array}$ & $\mathrm{x} 2$ & $\mathrm{x} 4$ & $\begin{array}{l}2.52 \mathrm{E} 6 \\
(\mathrm{x} 1.9)\end{array}$ & $\begin{array}{c}4.50 \mathrm{E} 6 \\
(\mathrm{x} 3.4)[\times 3.7]\end{array}$ \\
\hline $\begin{array}{l}\text { Standalone C++ "avx2" } \\
\text { [-march=haswell] }\end{array}$ & $\mathrm{x} 4$ & $\mathrm{x} 8$ & $\begin{array}{l}4.58 \mathrm{E} 6 \\
(\times 3.5)\end{array}$ & $\begin{array}{c}8.17 \mathrm{E} 6 \\
(\mathrm{x} 6.2)[x 6.8]\end{array}$ \\
\hline $\begin{array}{l}\text { Standalone C++ "512y" } \quad \text { (256-bit AVX512) } \\
{[- \text { march=skylake-avx512 -mprefer-vector-width=256] }}\end{array}$ & $\mathrm{x} 4$ & $\mathrm{x} 8$ & $\begin{array}{l}4.91 \mathrm{E} 6 \\
(\mathrm{x} 3.7)\end{array}$ & $\begin{array}{c}8.84 \mathrm{E} 6 \\
(\mathrm{x} 6.7)[x 7.3]\end{array}$ \\
\hline $\begin{array}{l}\text { Standalone C++ "512z" (512-bit AVX512) } \\
\text { [-march=skylake-avx512 -DMGONGPU_PVW512] }\end{array}$ & $\mathrm{x} 8$ & $\mathrm{x} 16$ & $\begin{array}{c}3.74 \mathrm{E} 6 \\
(\mathrm{x} 2.9)\end{array}$ & $\begin{array}{c}7.42 \mathrm{E} 6 \\
(\times 5.7)[x 6.1]\end{array}$ \\
\hline
\end{tabular}

\begin{tabular}{|lc|c|c|c|c|}
\hline GPU build name $\left(e^{+} e^{-} \rightarrow \mu^{+} \mu^{-}\right)$ & \multicolumn{2}{|c|}{ MEs/sec (compute) } & \multicolumn{2}{|c|}{ MEs/sec (compute + DtoH $)$} \\
\cline { 3 - 6 } GPU model & $($ Theoretical TFlops $)$ & Double & Float & Double & Float \\
\hline \hline Standalone CUDA & & $1.37 \mathrm{E} 9$ & $3.28 \mathrm{E} 9$ & $7.25 \mathrm{E} 8$ & $1.59 \mathrm{E} 9$ \\
NVidia V100 & $(7.1 /$ double, 14.1/float $)$ & $(\mathrm{x} 1050)$ & $(\mathrm{x} 2500)$ & $(\mathrm{x} 550)$ & $(\mathrm{x} 1200)$ \\
\hline Standalone CUDA & $4.01 \mathrm{E} 7$ & $8.22 \mathrm{E} 8$ & $3.89 \mathrm{E} 7$ & $6.38 \mathrm{E} 8$ \\
NVidia T4 & $(0.25 /$ double, $8.1 /$ float $)$ & $(\mathrm{x} 31)$ & $(\mathrm{x} 630)$ & $(\mathrm{x} 30)$ & $(\mathrm{x} 490)$ \\
\hline
\end{tabular}

Table 2. Preliminary results. Throughput of the ME calculation for $e^{+} e^{-} \rightarrow \mu^{+} \mu^{-}$, in Fortran, $\mathrm{C}++$ and CUDA, using double or single precision floating point arithmetics. For Fortran: estimate from MATRIX1 in MadEvent. For C++ and CUDA: measurements from the standalone executables, over 12 iterations with 524k events (2048 blocks, 256 threads per block in CUDA). Compilers: gcc9.2 and CUDA11.0. All builds use "-O3", and either "-ffast-math" (C++) or "-use_fast_math" (CUDA). Virtual machines itscrd70 (Fortran, C++ and CUDA/V100 results) and lxplus770 (CUDA/T4 results), both using skylake-avx512 CPUs (Intel Xeon Silver 4216) with 4 virtual cores. Fortran and C++ throughputs use a single CPU core. The rightmost CUDA throughputs include device-to-host copies of all ME values. Theoretical TFlops values for Nvidia V100 [45] and T4 [55] from TechPowerUp [56, 57].

It was thus quite natural to also develop an implementation of SIMD (Single Instruction Multiple Data) vectorization in our $\mathrm{C}++$ code for CPUs, similarly based on event-level data parallelism. In practice, the idea is that the innermost loop must be the loop over events, and more specifically over the contiguous events in one AOSOA page, so that each low-level arithmetic operation (e.g. a sum) on a pair of floating point numbers can be simultaneously performed on several pairs of numbers as a single SIMD instruction. This has required several disruptive changes to the code, which had not been needed to achieve event-level data parallelism in CUDA. A first specific example is that the loop over helicities, which in the initial CUDA implementation was repeated in each event, had to be moved outside the event loop. This also required some modification in the algorithm that determines which helicity combinations are valid. A second example is that the interfaces of IXXXXX, OXXXXX and FFV functions had to be modified to deal with input and output data describing several events, instead of individual events, and that their internal implementations had to be changed so that arithmetic operations manipulate vector data instead of scalar data. Both of these goals were achieved by defining new vector data types, e.g. fptype_v for a vector of floats or doubles, using the gcc [53] or clang [54] compiler vector extensions. A vector of complex numbers, cxtype_v, is defined as a SOA where the real and imaginary parts are separately stored in two contiguous fptype_v arrays (RRRRIIII), rather than as an AOS (RIRIRIRI). An additional type cxtype_sv is a scalar in CUDA and a vector in C++, so that, similarly, a "+" sign indicates a scalar sum in CUDA and a vector sum in $\mathrm{C}++$; the power of this approach is that the interface and implementation code of FFV functions are formally identical for CUDA and $\mathrm{C}++$, which considerably simplifies the backport to the code-generating Python metacode. 
Our C++ implementation of the ME calculation supports different SIMD architectures and can currently be built in five modes. These are summarized in Tab. 2, with the corresponding preliminary throughputs achieved on an Intel Xeon Silver 4216 CPU. In our baseline gcc implementation, the best results are achieved in the " $512 \mathrm{y}$ " build, which uses AVX512 with vector widths limited in our source code to 256 bits. In this case, where each fptype_v is an array of 4 doubles or 8 floats, throughput increases of x3.7 and x7.3 are achieved in double and single precision, compared to the corresponding scalar builds. These throughputs are 5\% to $10 \%$ higher than those achieved using the same vector dimensions in the AVX2 build; disassembly using objdump indicates that this is thanks to a few additional vector instructions on the 256-bit ymm registers, introduced in the AVX512VL extensions. Further analyses and optimisations are in progress on the " $512 z$ " build, based on AVX512 with default 512-bit zmm registers, which could theoretically deliver a higher throughput because it uses vectors of 8 doubles or 16 floats, but has so far achieved a worse throughput than AVX2.

In our C++ implementation, the striding of 4-momenta AOSOA's is presently hardcoded to the dimension of fptype_v arrays. This is meant to ensure that vectorization can lead to throughput increases not only in floating point operations (e.g. because a single AVX2 instruction executes 4 sums of doubles simultaneously), but also in data access from memory (e.g. because a single AVX2 instruction loads the $e^{+}$energies for 4 different events). Work is in progress on a more flexible implementation, which will allow a detailed performance analysis of these two separate effects, and will also simplify the integration of the CUDA/C++ ME calculation in the Fortran MadEvent infrastructure, discussed in Sec. 5.

SIMD vectorization and multi-core execution are two largely independent concurrency mechanisms that should not be confused, although they can both be exploited through eventlevel parallelism. The above description of the $\mathrm{C}++$ implementation and the results in Tab. 2 all refer to a single CPU core. Preliminary tests [13] on a few multi-core CPUs show that ME throughputs scale well, up to the number of physical cores and also in the hyper-threading regime, if several copies of our standalone $\mathrm{C}++$ application are executed simultaneously. A better option is to use multithreading (MT) to process different sets of events in separate $\mathrm{C}++$ threads, as this has the advantage of reducing the total memory footprint on a node. Our first MT implementation, based on OpenMP [58], is not yet delivering a stable and satisfactory performance, and needs further optimizations. Eventually, a heterogeneous ME engine can also be developed, where the ME computation runs for some events in $\mathrm{C}++$ on different $\mathrm{CPU}$ threads, while for other events it is offloaded to the GPU via CUDA. A basic prototype of this functionality already exists, but needs further design and optimisation efforts.

\section{Summary of preliminary results, future plans and outlook}

A summary of our preliminary results is given in Tab. 2, in terms of the throughputs (in MEs per second) of the $e^{+} e^{-} \rightarrow \mu^{+} \mu^{-}$ME calculation on two test systems.

The throughputs achieved by our $\mathrm{C}++$ and CUDA ME engines have already been partially discussed in the previous section. Compared to a scalar $\mathrm{C}++$ implementation of the ME calculation, software vectorization using AVX512 and 256-bit wide registers achieves a throughput increase by factors which are only marginally lower than the $\mathrm{x} 4$ and $\mathrm{x} 8$ theoretically achievable in double and single precision. Neglecting the time needed for the deviceto-host copies of MEs, the throughputs achieved on a full V100 GPU are larger than that of scalar $\mathrm{C}++$ on a single CPU core by factors of approximately x1000 and x2500 in double and single precision. This large difference is mainly due to the fact that a V100 [45] has twice as many FP32 cores (5120) as FP64 cores (2560), thus delivering a theoretical performance in single precision (14.1 TFlops) which is twice that in double precision (7.1 TFlops). The lower register pressure of SIGMAKIN in single precision, previously discussed, also contributes an additional speedup. The situation is very different on a T4 [55] GPU, where we observe speedups of approximately x30 and x600 for double and float compared to our scalar 
$\mathrm{C}++$ code; the much lower performance of the $\mathrm{T} 4$ in single precision is due to the much larger difference between the maximum theoretical performances [57] of this GPU for FP64 (0.25 TFlops) and FP32 (8.1 TFlops), which are in a ratio 1:32. We expect that newer generations of Nvidia data center GPUs will probably look more similar to the V100 than the T4, with a 1:2 ratio between FP64 and FP32 performances: for instance, this is the case of the A100 [59], with theoretical peak performances of 9.7 and 19.5 TFlops, respectively.

In other words, both for $\mathrm{C}++$ and CUDA, even if for very different reasons, using single precision instead of double precision floating point arithmetics generally results in at least a factor $\mathrm{x} 2$ throughput increase (and much more on some GPUs). This option should therefore be carefully considered if additional large speedups are needed. This choice, however, would require an important effort to rewrite some calculations for a better control of numeric instabilities, followed by a careful validation of physics results; even in the simple $e^{+} e^{-} \rightarrow \mu^{+} \mu^{-}$ baseline use case, we have observed that in around one event in one million the calculated ME takes a Not-a-Number $(\mathrm{NaN})$ value if single precision is used, both for CUDA and for $\mathrm{C}++$.

In addition to the CUDA/C++ ME throughputs measured in our RAMBO-based standalone $\mathrm{C}++$ application, Tab. 2 includes a rough estimate of the throughput for the ME calculation in the production Fortran implementation of MG5aMC, where phase space sampling is driven by MadEvent. Using similar compilation options (in particular, fast math), our scalar $\mathrm{C}++$ implementation on a single CPU core seems to be slower than the corresponding Fortran version by only $15 \%$. We stress that this is not an apple-to-apple comparison, but we believe that this gives a reasonable order of magnitude of the throughput gains that could be achieved by using our CUDA/C++ implementation instead of Fortran for the ME calculation.

Our current plan, in particular, which we have already discussed with the LHC experiments [60], consists in injecting the new CUDA/C++ implementation of the ME calculation into the existing, MadEvent-based, MG5aMC infrastructure. This is a complex mix of Fortran, Python and shell scripts, which the LHC experiments have thoroughly validated and routinely use for cross section calculations and unweighted event generation. We therefore believe that the fastest way to bring our new developments to production consists in reusing most of this existing infrastructure, and in particular its outer shell with its well-documented user interface, while only replacing its inner core that represents its computational bottleneck. Amongst other things, this strategy has the advantage that many complex software and physics issues, such as event I/O to files or the interface to PDFs and parton showers, should automatically be taken care of by the existing MG5aMC. Many functionalities will however require further work, such as the interface to loop libraries required for NLO calculations.

In practice, this strategy mainly requires the reengineering of the Fortran code that within MadEvent calls the ME calculation function for a given event, from the 4-momenta of its particles. In particular, the event loops must be modified so that several phase MEs are computed in parallel for a set of pre-prepared phase space points, and special care must also be taken to identify all relevant inputs and outputs that the current ME calculation in Fortran processes through function arguments or, more often, Fortran common blocks. Care must also be taken in the way 4-momenta data is passed from Fortran to $\mathrm{C}++$, although the overhead of suboptimal data loading is expected to become negligible as the complexity of the ME calculation increases. Work in this area has already started and is making rapid progress.

It should be stressed that the throughput increase factors quoted above do not refer to a full software workflow for calculating cross sections or generating weighted or unweighted events, but only to the component responsible for the ME calculation. Amdahl's law [61, 62] puts stringent constraints on the achievable increase in the throughput of the full software workload, if a significant fraction of it cannot be parallelized. Taking into account, however, that the ME calculation often accounts for much more than $90 \%$ of the total CPU time, overall speedups by a factor $3 \mathrm{x}$ or more on a CPU through vectorization seem to be within reach.

While our focus is shifting to the integration of Fortran and CUDA/C ++ as described above, we will keep using the simplified CUDA/C++ standalone executable as our workhorse 
for all ME optimisations. We also plan to continue prototyping some functional enhancements of this application, albeit at a much lower priority. This ranges from the implementation of proper cross section calculations and event unweighting, to improvements in phase space sampling (through the vectorization of RAMBO, the reimplementation of MadEvent in CUDA/C ++ or the use of external packages such as VegasFlow [63]), to the integration of parton distribution functions (possibly through external packages such as PDFFlow [64]). In this context, we also closely follow the related developments by the MadFlow team $[65,66]$.

Finally, we believe that our implementations of ME calculations in CUDA (and eventually other languages) for GPUs and in $\mathrm{C}++$ for vector CPUs represent very useful software workloads for the benchmarking of computing resources used by the HEP experiments, in an increasingly heterogeneous environment. In this context, we are collaborating with the HEPiX benchmarking working group, with the goal of eventually providing a MG5aMC-based containerized standalone application to be integrated in the HEP-Benchmarks suite [67, 68]. We also plan to port our code to new architectures, such as AMD and Intel GPUs but also non-x86 CPUs such as ARM and Power9, also in view of the use of the software at HPC facilities.

\section{Conclusions}

In this paper, we have presented an ongoing project aiming at reengineering the MG5aMC physics event generator, primarily to develop a new CUDA back-end for GPUs, but also to optimize the performance of its $\mathrm{C}++$ back-end on CPUs. We have described its motivation, its iterative engineering process involving code-generating metacode, its software architecture based on event-level data parallelism to efficiently exploit SIMT on GPUs and SIMD on CPUs, as well as some current challenges and future directions. We have also summarized the preliminary results that we have presented at the vCHEP2021 conference [13], providing an update over previous preliminary results shown in the past [69]. Our first results are very encouraging, showing that speedups of the ME calculation by more than a factor 3 on CPUs are possible through SIMD vectorization, and by three orders of magnitude on a typical data center GPU. We are now focusing on a strategy to bring these improvements to a production release of the code usable by the LHC experiments in a relatively short time, by injecting our new CUDA/C++ component into the existing Fortran-based infrastructure of MG5aMC. This is work in progress, about which further updates will be presented in later publications.

\section{Acknowledgements}

It is a pleasure to thank our colleagues in the madgraph4gpu project, Tyler Burch, Taylor Childers, Smita Darmora, Laurence Field, Walter Hopkins, Josh McFayden, Vince Pascuzzi, Markus Schulz, David Smith and Carl Vuosalo, for our fruitful collaboration, and Andy Reepschlaeger and Taran Singhania also for their contributions to the infrastructure for the analysis of performance metrics. We are grateful to Sebastien Ponce, Hadrien Grasland and Marco Clemencic for many suggestions about SIMD vectorization, and to Ricardo Rocha and CERN IT-CM for their help in setting up our GPU build nodes. We thank Domenico Giordano and the HEPIX benchmarking working group, as well as Helge Meinhard and the WLCG benchmarking task force, for stimulating discussions on benchmarking of GPUs and heterogeneous systems. Useful discussions with Stefano Carrazza and the MadFlow team, as well as with Ingvild Brevik Hoegstoeyl and her colleagues in NTNU and OpenLab, are gratefully acknowledged. We thank the authors of the MG5aMC software for their continued support.

We are indebted to the organizers of the Sheffield GPU Hackathon [70]. We particularly wish to thank Andreas Herten, Peter Heywood and Mateusz Malenta for the support they provided as our mentors and their many useful suggestions about our CUDA development. 


\section{References}

[1] J. Alwall et al., The automated computation of tree-level and next-to-leading order differential cross sections, and their matching to parton shower simulations, JHEP07(2014)079. https://doi.org/10.1007/JHEP07(2014)079

[2] A. Valassi, E. Yazgan, J. McFayden (eds.) et al., Challenges in Monte Carlo event generator software for High-Luminosity LHC, Comput. Softw. Big Sci. 5, 12 (2021). https://doi.org/10.1007/s41781-021-00055-1

[3] A. Valassi, E. Yazgan, J. McFayden, Monte Carlo generator strategy towards HL-LHC, WLCG meeting with LHCC referees (2020). https://doi.org/10.5281/zenodo.4028834

[4] Nvidia, CUDA Toolkit. https://developer.nvidia.com/cuda-toolkit

[5] K. Hagiwara et al., Fast calculation of HELAS amplitudes using graphics processing unit (GPU), Eur. Phys. J. C 66 (2010) 477. https://doi.org/10.1140/epjc/s10052-010-1276-8

[6] K. Hagiwara et al., Calculation of HELAS amplitudes for QCD processes using graphics processing unit (GPU), Eur. Phys. J. C 70 (2010) 513. https://doi.org/10.1140/epjc/s10052-010-1465-5

[7] K. Hagiwara et al., Fast computation of MadGraph amplitudes on graphics processing unit (GPU), Eur. Phys. J. C 73 (2013) 2608. https://doi.org/10.1140/epjc/s10052-013-2608-2

[8] J. Kanzaki, Monte Carlo integration on GPU, Eur. Phys. J. C 71 (2011) 1559. https://doi.org/10.1140/epjc/s10052-011-1559-8

[9] J. Kanzaki, Application of graphics processing unit (GPU) to software in elementary particle/high energy physics field, Procedia Computer Science 4 (2011) 869. https://doi.org/10.1016/j.procs.2011.04.092

[10] “MadGraph5_aMC@NLO on GPU” project. https://madgraph5.github.io

[11] HSF Physics Event Generator Working Group. https://hepsoftwarefoundation.org/workinggroups/generators.html

[12] 25th International Conference on Computing in High-Energy and Nuclear Physics (vCHEP2021), 17-21 May 2021. https://indico.cern.ch/event/948465

[13] A. Valassi, Reengineering the MadGraph5_aMC@NLO Monte Carlo event generator for GPUs and vector CPUs, talk presented at vCHEP2021 (2021). https://doi.org/10.5281/zenodo.4785174 and https://doi.org/10.17181/CERN.ESFS.PYDP

[14] S. Frixione, B. R. Webber, Matching NLO QCD computations and parton shower simulations, JHEP06(2002)029. https://doi.org/10.1088/1126-6708/2002/06/029

[15] J. Alwall, M. Herquet, F. Maltoni, O. Mattelaer, T. Stelzer, MadGraph 5: going beyond, JHEP06(2011)128. https://doi.org/10.1007/JHEP06(2011)128

[16] O. Mattelaer, K. Ostrolenk, Speeding up MadGraph5_aMC@NLO, MCNET-21-01 (2021). arxiv:2102.00773

[17] C. Degrande et al., UFO - the Universal FeynRules output, Comp. Phys. Comm. 183 (2012) 1201. https://doi.org/10.1016/j.cpc.2012.01.022

[18] A. Alloul, N. D. Christensen, C. Degrande, C. Duhr, B. Fuks, FeynRules 2.0 - A complete toolbox for tree-level phenomenology, Comp. Phys. Comm. 185 (2014) 2250. https://doi.org/10.1016/j.cpc.2014.04.012

[19] A. Semenov, LanHEP - A package for automatic generation of Feynman rules from the Lagrangian. Version 3.2, Comp. Phys. Comm. 201 (2016) 167. https://doi.org/10.1016/j.cpc.2016.01.003

[20] F. Staub, SARAH (2008). arxiv:0806.0538

[21] F. Maltoni, T. Stelzer, MadEvent: automatic event generation with MadGraph, JHEP02(2003)027. https://doi.org/10.1088/1126-6708/2003/02/027

[22] R. Kleiss, The cross section for $e^{+} e^{-} \rightarrow e^{+} e^{-} e^{+} e^{-}$, Nucl. Phys. B 241 (1984) 61. https://doi.org/10.1016/0550-3213(84)90197-4 
[23] K. Hagiwara, D. Zeppenfeld, Helicity amplitudes for heavy lepton production in $e^{+} e^{-}$ annihilation, Nucl. Phys. B 274 (1986) 1. https://doi.org/10.1016/0550-3213(86)90615-2

[24] K. Hagiwara, D. Zeppenfeld, Amplitudes for multi-parton processes involving a current at $e^{+} e^{-}, e^{ \pm} p$ and hadron colliders, Nucl. Phys. B 313 (1989) 560.

https://doi.org/10.1016/0550-3213(89)90397-0

[25] P. de Aquino, W. Link, F. Maltoni, O. Mattelaer, T. Stelzer, ALOHA: Automatic libraries of helicity amplitudes for Feynman diagram computations, Comp. Phys. Comm. 183 (2012) 2254. https://doi.org/10.1016/j.cpc.2012.05.004

[26] F. Maltoni, K. Paul, T. Stelzer, S. Willenbrock, Color-flow decomposition of QCD amplitudes, Phys. Rev. D 67 (2003) 014026. https://doi.org/10.1103/PhysRevD.67.014026

[27] F. Halzen, A. D. Martin, Quarks and leptons: an introductory course in modern particle physics, Wiley (1984).

[28] J. P. Ellis, TikZ-Feynman: Feynman diagrams with TikZ, Comp. Phys. Comm. 210 (2017) 103. https://doi.org/10.1016/j.cpc.2016.08.019

[29] H. Murayama, I. Watanabe, K. Hagiwara, HELAS: HELicity Amplitude Subroutines for Feynman Diagram Evaluations, KEK-Report 91-11 (1992). https://lib-extopc.kek.jp/preprints/PDF/1991/9124/9124011.pdf

[30] I. Watanabe, H. Murayama, K. Hagiwara, Evaluating Cross Sections at TeV Energy Scale by HELAS, KEK preprint 92-39 (1992). https://lib-extopc.kek.jp/preprints/PDF/1992/9227/9227039.pdf

[31] AMD, ROCm documentation: HIP Programming Guide. https://rocmdocs.amd.com/en/latest/Programming_Guides/HIP-GUIDE.html

[32] E. Zenker et al., Alpaka - An Abstraction Library for Parallel Kernel Acceleration, Proc. IEEE IPDPSW 2016, Chicago. https://doi.org/10.1109/IPDPSW.2016.50

[33] Exascale Computing Project, Kokkos ecosystem. https://kokkos.org

[34] Khronos Group, SYCL. https://www.khronos.org/sycl

[35] “MadGraph5_aMC@NLO on GPU” project, madgraph4gpu code repository. https://github.com/madgraph5/madgraph4gpu

[36] “MadGraph5_aMC@NLO on GPU” project, madgraph4gpu code tag CHEP2021. https://doi.org/10.5281/zenodo.5087381

[37] MadGraph5_aMC@NLO, official code repository. https://launchpad.net/mg5amcnlo

[38] Nvidia, cuRAND: the CUDA random number generation library. https://developer.nvidia.com/curand

[39] R. H. Kleiss, W. J. Stirling, S. D. Ellis, A new Monte Carlo treatment of multiparticle phase space at high energies, Comp. Phys. Comm. 40 (1986) 359. https://doi.org/10.1016/0010-4655(86)90119-0

[40] Google, GoogleTest. https://github.com/google/googletest

[41] E. Boos et al., Generic User Process Interface for Event Generators, Proc. Physics at TeV colliders Workshop, Les Houches (2001). arxiv:hep-ph/0109068

[42] J. Alwall et al., A standard format for Les Houches Event Files, Comp. Phys. Comm. 176 (2007) 300. https://doi.org/10.1016/j.cpc.2006.11.010

[43] T. Sjöstrand et al., An introduction to PYTHIA 8.2, Comp. Phys. Comm. 191 (2015) 159. https://doi.org/10.1016/j.cpc.2015.01.024

[44] S. Agostinelli et al., Geant4 - a simulation toolkit, Nucl. Instr. Meth. A 506 (2003) 250. https://doi.org/10.1016/S0168-9002(03)01368-8

[45] Nvidia, Nvidia Tesla V100 GPU architecture. https://images.nvidia.com/content/voltaarchitecture/pdf/volta-architecture-whitepaper.pdf

[46] Nvidia, Nsight Systems. https://developer.nvidia.com/nsight-systems

[47] Nvidia, CUDA C++ Best Practices Guide: Coalesced Access to Global Memory. https://docs.nvidia.com/cuda/cuda-c-best-practices-guide/index.html\#coalesced-accessto-global-memory 
[48] Nvidia, Nsight Compute. https://developer.nvidia.com/nsight-compute

[49] Nvidia, Nsight Compute CLI user manual: Nvprof Transition Guide, Metric Comparison. https://docs.nvidia.com/nsight-compute/NsightComputeCli/index.html\#nvprofmetric-comparison

[50] Nvidia, CUDA C++ Programming Guide: Technical Specifications per Compute Capability. https://docs.nvidia.com/cuda/cuda-c-programming-guide/index.html\#features-andtechnical-specifications

[51] Nvidia, CUDA C++ Programming Guide: CUDA Graphs. https://docs.nvidia.com/cuda/cuda-c-programming-guide/index.html\#cuda-graphs

[52] Nvidia, CUDA C++ Programming Guide: SIMT architecture. https://docs.nvidia.com/cuda/cuda-c-programming-guide/index.html\#simt-architecture

[53] GNU Compiler Collection documentation, Using Vector Instructions through Built-in Functions. https://gcc.gnu.org/onlinedocs/gcc/Vector-Extensions.html

[54] Clang documentation, Clang Language Extensions: Vectors and Extended Vectors. https://clang.llvm.org/docs/LanguageExtensions.html\#vectors-and-extended-vectors

[55] Nvidia, Nvidia Turing GPU architecture. https://www.nvidia.com/content/dam/enzz/Solutions/design-visualization/technologies/turing-architecture/NVIDIA-TuringArchitecture-Whitepaper.pdf

[56] TechPowerUp GPU database, Nvidia Tesla V100 PCIe 32 GB. https://www.techpowerup.com/gpu-specs/tesla-v100-pcie-32-gb.c3184

[57] TechPowerUp GPU database, Nvidia Tesla T4 Specs. https://www.techpowerup.com/gpu-specs/tesla-t4.c3316

[58] The OpenMP API specification for parallel programming, https://www.openmp.org

[59] Nvidia, Nvidia A100 Tensor Core GPU architecture. https://images.nvidia.com/aemdam/en-zz/Solutions/data-center/nvidia-ampere-architecture-whitepaper.pdf

[60] S. Roiser, MG5aMC plans for GPUs and vectorization, HSF generator WG meeting (May 2021). https://hepsoftwarefoundation.org/organization/2021/05/06/generators.html

[61] Wikipedia, Amdahl's law. https://en.wikipedia.org/wiki/Amdahl\%27s_law

[62] G. M. Amdahl, Validity of the Single Processor Approach to Achieving LargeScale Computing Capabilities, AFIPS Conference Proceedings (30): 483-485. https://doi.org/10.1145/1465482.1465560

[63] S. Carrazza, J. M. Cruz-Martinez, VegasFlow: accelerating Monte Carlo simulation across multiple hardware platforms, Comp. Phys. Comm. 254 (2020) 107376. https://doi.org/10.1016/j.cpc.2020.107376

[64] S. Carrazza, J. M. Cruz-Martinez, M. Rossi, PDFFlow: parton distribution functions on GPU (2020). arxiv:2009.06635

[65] J. M. Cruz-Martinez, MadFlow: towards the automation of Monte Carlo simulation on GPU for particle physics processes, talk presented at vCHEP2021 (2021). https://indico.cern.ch/event/948465/contributions/4324113

[66] S. Carrazza, J. M. Cruz-Martinez, M. Rossi, M. Zaro, Towards the automation of Monte Carlo simulation on GPU for particle physics processes, to appear in Proc. vCHEP2021. arxiv:2105.10529

[67] A. Valassi et al., Using HEP experiment workflows for the benchmarking and accounting of WLCG computing resources, Proc. CHEP2019, EPJ Web of Conf. 245, 07035 (2020). https://doi.org/10.1051/epjconf/202024507035

[68] Miguel Fontes Medeiros, HEPiX benchmarking solution for WLCG computing resources, talk presented at vCHEP2021 (2021).

https://indico.cern.ch/event/948465/contributions/4323674

[69] S. Roiser, Progress on porting MadGraph5_aMC@NLO to GPUs, HSF/WLCG Virtual Workshop (2020). https://indico.cern.ch/event/941278/contributions/4101793

[70] Sheffield Virtual GPU Hackathon 2020. https://gpuhack.shef.ac.uk 